\title{
Energy-dissipation in a coupled system of Allen-Cahn type equation and Kobayashi-Warren-Carter type model of grain boundary motion
}

\author{
Hiroshi Watanabe $^{1}$ and Ken Shirakawa ${ }^{2}$ \\ ${ }^{1}$ Oita University \\ ${ }^{2}$ Chiba University
}

May 5, 2020

\begin{abstract}
In this paper, we consider a system of initial boundary value problems for parabolic equations, as a generalized version of the " $\varphi-\eta-\vartheta$ model" of grain boundary motion, proposed by Kobayashi [16]. The system is a coupled system of: an AllenCahn type equation as in (1.1) with a given temperature source; and a phase-field model of grain boundary motion, known as "Kobayashi-Warren-Carter type model". The focus of the study is on a special kind of solution, called energy-dissipative solution, which is to reproduce the energy-dissipation of the governing energy in time. Under suitable assumptions, two Main Theorems, concerned with: the existence of energy-dissipative solution; and the large-time behavior; will be demonstrated as the results of this paper.
\end{abstract}

\section{Hosted file}

WS05.pdf available at https://authorea.com/users/302395/articles/432460-energy-dissipationin-a-coupled-system-of-allen-cahn-type-equation-and-kobayashi-warren-carter-type-modelof-grain-boundary-motion 